\title{
COMUNIDADES ALGALES DEL CABO DE CREUS (ENTRE LOS 0 Y 100 M. DE ALTITUD) Y SU RELACION CON EL AMBIENTE
}

\author{
X. Tomas*, J. Cambra** y S. Sabater* \\ *Dpto. de Ecología. Fac. de Biología. Univ. de Barcelona. Av. Diagonal, 645. 08028 B ARCELONA \\ ** Dpto. de Botánica. Fac. de Biología. Univ. de Barcelona. Av. Diagonal, 645. 08028 B ARCELONA.
}

Palabras clave: Limnology, algal communities, Cape of Creus, phytosociology.

\author{
ABSTRACT \\ ALGAL COMMUNITIES OFCAPE CREUS (BETWEEN 0 AND 100 M. A.S.L.) AND THEIR RELATION \\ WITH THE ENVIRONMENT
}

Cape Creus is a small peninsula $\left(120 \mathrm{~km}^{2}\right)$ located in the NE of Spain. It is a mountainous region formed by silicic materials (schists, gneisses and granites) of paleozoic origin (Cambrian-Ordovician).

In this paper the results of the limnological study of the litoral band $(0-100 \mathrm{~m}$, a.s.l. are presented. A total of 12 aquatic systems were seasonally sampled during 1984 . All of them are temporary creeks running into the sea, being shallow and having a small flow, variable slope and a large marine influence. The $\mathrm{pH}$ is acid in february (about 6.5) and rather basic in the rest of the year. The alkalinity is moderate (about $1 \mathrm{meq} / 1$ ), and the conductivity high with a mean value of $1147 \mathrm{uS} / \mathrm{cm}$ and a standard deviation of 839 $\mathrm{uS} / \mathrm{cm}$.

In those stations closest to the sea the conductivity is the most fluctuant parameter. The maxima are in september and february. These fluctuations are caused mainly by aerial transport of marine salts. In the rest of stations all parameters are more stable. Only the alkalinity shows an important maximum in september, which can be explained by the summer decomposition of organic matter (mainly filamentous algae).

From a phytosociological point of view, the algal communities belong to the Tribonemetalia dominium. The Melosiretum rivulare and Hildenbrandietum rivulure grow on rocky substrates with fast water flow. In deep small basins where water current is slower the plocon is formed mainly by the associations of the Al. Tribonemion. The diatom communities may belong to these associations, but usually constitute a Melosiretum fluviatile. In places with strong marine influence some species ofthe Al. Synedrion tabulatae are the most important. In all stations there is a common pool of halophile and aerophilic species which indicates the excess of clonde and the temporary nature ofthe systems studied.

Most communities change in summer: Melosiretum rivulare dissapears, Tribonemion decays and among diatoms species characteristic of eutrophic waters with much decomposing organic matter (Nav. cryptocephala, Nitzschia sp. pl., etc.) predominate. On the other hand, some elements of the Cladophorion (Rhizoclonium hieroglyphicum, Cladophora crussior) appear or extend their ranges.

In autumn the communities regenerate, and the species characteristic of the initial facies of the corresponding associations appear again.

\section{INTRODUCCION}

El Cabo de Creus ha sido considerado, al menos en los últimos tiempos, como uno de los lugares de Ca-

Limnetica 3: 111-1 19 (1987)

○ Asociación Española de Limnologia, Madrid. Spain taluña más atractivos para los naturalistas locales. Paradójicamente, la literatura dedicada a los distintos aspectos de su biología es muy escasa. Basta comprobar, por ejemplo, que no existe monografía alguna re- 


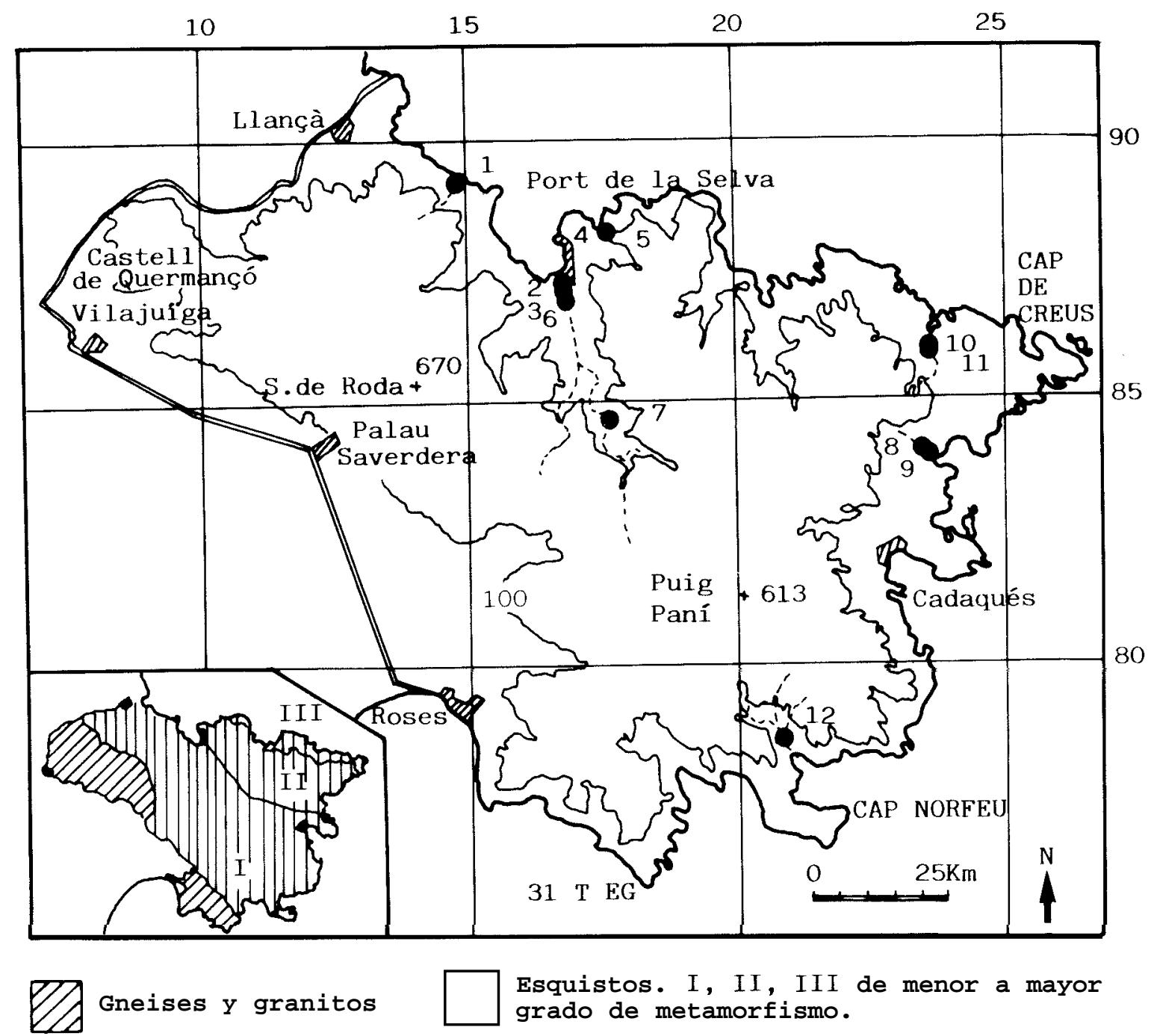

Figura 1.-Distribución de las estaciones en el área de estudio. En la parte inferior izquierda se indican las principales regiones limnológicas.

Distribution ofthe stations in the study area. The main litological regions are shown at the bottom left.

ferida al estudio de las algas continentales de la región. Los únicos datos disponibles son breves comentarios, que aparecen en varias notas de MARGALEF $(1949,1950,1953,1954,1955$ y 1958) sobre la hidrobiología del NE de España.

Esta falta de información y la reciente propuesta de convertir esta zona en Parque Natural, motivaron el inicio de un programa de investigación pluridisciplinario, denominado «Flora i vegetació criptogámica del proposat Parc Natural del Cap de Creus. I. Zona costanera)), que se ha desarrollado a lo largo de 1984 , con la subvención de la Universidad de Barcelona.
En las líneas que siguen, se presentan una parte de los resultados obtenidos. Se describen las asociaciones de algas de los ambientes acuáticos situados entre el nivel del mar y los $100 \mathrm{~m}$ de altitud, se comenta su evolución temporal y su relación con algunos parámetros ambientales.

\section{MATERIAL Y METODOS}

El Cabo de Creus esta situado en el extremo NE de la provincia de Gerona. Es una pequeña península, 


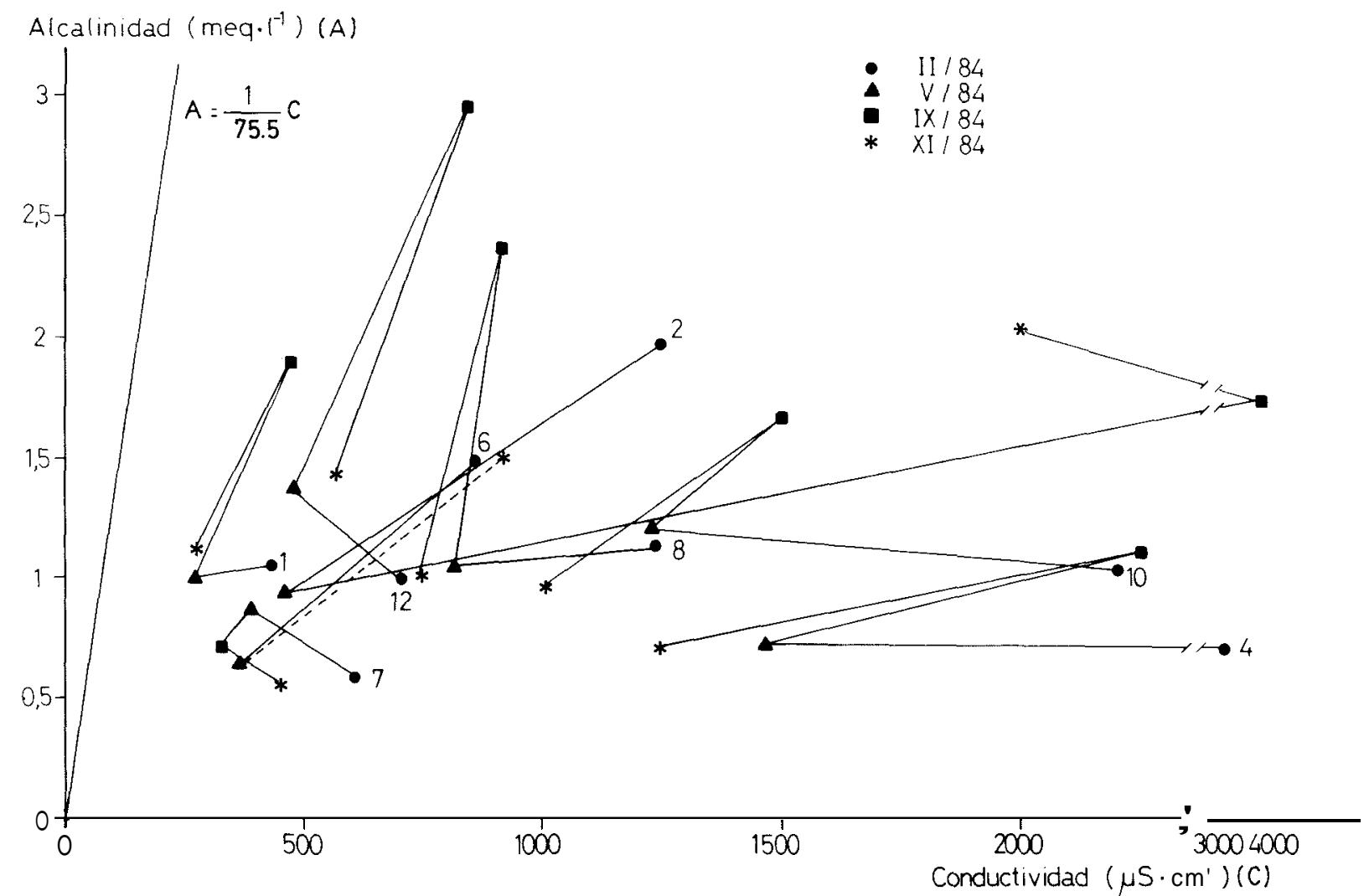

Figura 2.-Variación conjunta de la conductividad (C) y la alcalinidad (A) del agua en ocho estaciones a lo largo del ciclo anual. $\mathrm{A}=(1 / 75.5) \mathrm{C}$ es la ecuación de la recta teórica, formada por los puntos de igual relación alcalinidad / conductividad de un agua dulce «armónica» a distintas concentraciones. Para calcularla se ha consideradoque la alcalinidad del agua es debida únicamentea los bicarbonatos, cuya contribución a la alcalinidad se indica en la tabla III. Compárense la posición y pendiente de esta recta con la de los puntos correspondientesa cada localidad y los segmentosque los unen, respectivamente.

Annual joint vanation of water conductivity (C)and alkalinity $(A)$ in 8 sites over the year. $A=(1 / 75.5) C$ is the equation of the theoretical rect, formed by points with the same alkalinity/conductivity ratio of an «harmonic» freshwater at different concentrations.

con una superficie aproximada de $120 \mathrm{~km} .{ }^{2}$. Su perfil es complicado y el relieve recortado por pequeños valles, que establecen una red hidrografica sinuosa y con importantes desniveles. En pocos kilómetros se puede descender de las cotas más altas, como la Sierra de Roda (670 m.) o el Puig Pani (613 m.), a la orilla del mar.

Geológicamente forma parte de las estribaciones más orientales de la Sierra de la Albera. Los materiales de la región pertenecen al Paleozoico (CámbricoOrdovicico). Fueron afectados por la orogenia alpina, causante del levantamiento de los Pirineos, pero las estructuras de deformación y los procesos petrogenéticos asociados, tienen un origen exclusivamente herciniano (CARRERAS, 1974). Desde el punto de vista litológico, se distingue una zona formada por gneises y granitos, y otra consituida por esquistos peliticos en distinto grado metamórfico (fig. 1). Sólo en la mitad este del Cabo Norfeu afloran rocas de naturaleza calcárea.

Las doce localidades escogidas para este estudio están situadas en la zona esquistosa y a lo largo de la franja costera objeto de nuestro interés (fig. 1). Todas ellas son torrentes o ramblas de circulación temporal, que en la zona se conocen con el nombre de «recs». Las localidades son, por orden de numeración:

1. Torrente sin toponimia. Junto a la fuente Clareta. Altitud: $40 \mathrm{~m}$.

2 y 3. Rec de Romanyá. Altitud: despreciable.

4 y 5 . Torrente sin toponimia. Al Este de la Cala Tamariua. Altitud: 10 m. y 2 m., respectivamente.

6. Rec de Romanya. Altitud: despreciable. 


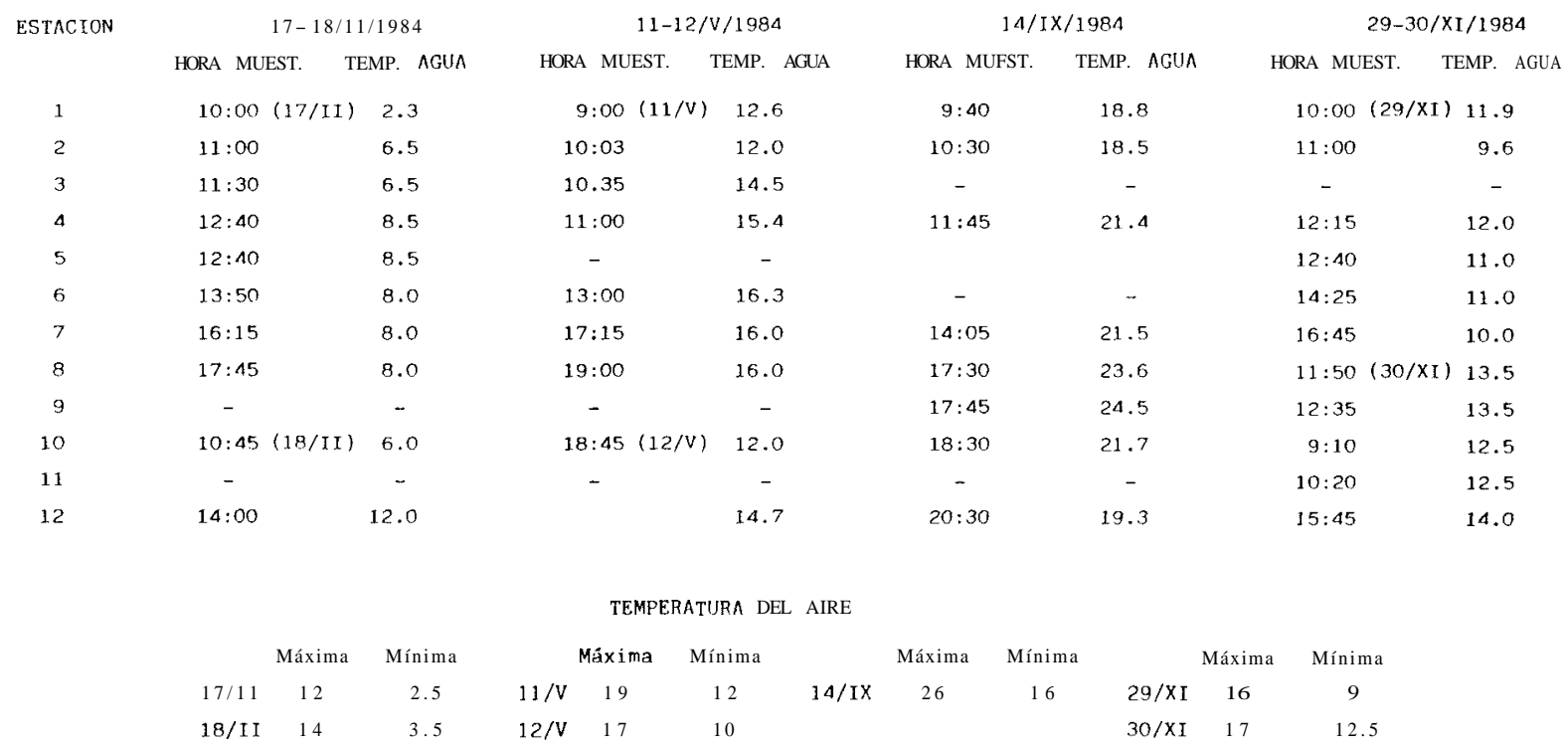

Tabla 1.-Variaciónde la temperatura del agua a lo largo del periodo de muestreo. Se indican las horas en que se efectuaron las medidas y los valores máximo y mínimo de la temperatura del aire en las fechascorrespondientes (datos del Obs. Meteor. de Port Bou). Water temperature variation through the study period. Monitoring time is given, as well as maximum and minimum air temperature of the day (data from Metheor. Obser. of Port Bou).

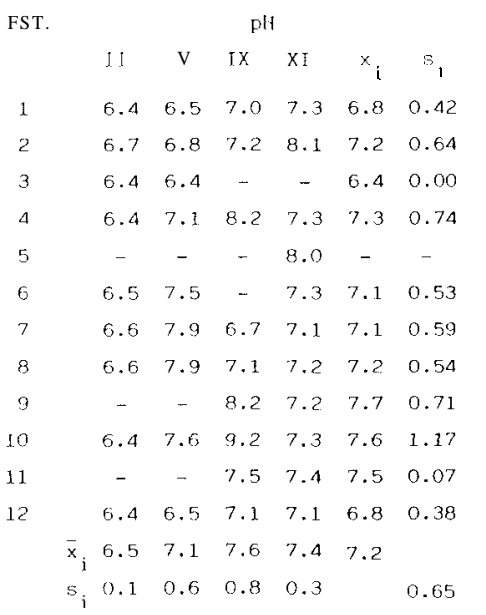

\begin{tabular}{cccccc}
\multicolumn{7}{c}{ CONDUCTIVIDAD } & \multicolumn{2}{c}{$(\mu \mathrm{s} / \mathrm{cm})$} \\
I I & $\mathrm{V}$ & \multicolumn{1}{c}{$\mathrm{IX}$} & \multicolumn{1}{c}{ XI } & \multicolumn{1}{c}{$\times_{\mathrm{i}}$} & 1 \\
435 & 270 & 475 & 277 & 364.3 & 106.1 \\
1242 & 454 & 4041 & 2000 & 1934.3 & 1539.8 \\
1407 & 375 & - & - & 891.0 & 729.7 \\
3056 & 1461 & 2250 & 1240 & 2001.8 & 825.8 \\
3740 & - & - & 1277 & 2508.5 & 1741.6 \\
865 & 369 & - & 905 & 713.0 & 298.6 \\
605 & 392 & 323 & 450 & 442.5 & 120.1 \\
1230 & 810 & 912 & 740 & 923.0 & 216.5 \\
- & - & 1261 & 780 & 1020.5 & 340.1 \\
2250 & 1226 & 1500 & 1000 & 1494.0 & 543.9 \\
- & - & 1442 & 1095 & 1268.5 & 245.4 \\
707 & 478 & 840 & 563 & 647.0 & 159.7 \\
1554 & 648 & 1449 & 939 & 1147.3 & \\
1110 & 426 & 1133 & 474 & & 892.1
\end{tabular}

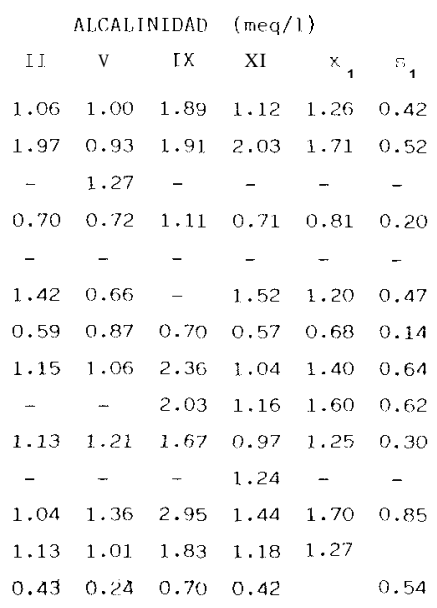

Tabla 2.-Variaciones del pH, conductividad y alcalinidad del agua a lo largo del periodo de estudio, indicándose las medias y desviaciones típicas por localidades $(\mathrm{x}, \mathrm{S}$,$) y épocas del año (\mathrm{x}, \mathrm{s}$,$) .$

Water $\mathrm{pH}$, conductivity and alkalinity variation through the study period. The means and standard deviations in sites $\left(\overline{\mathrm{x}}_{\mathrm{j}}, \mathrm{s}_{\mathrm{j}}\right)$ and periods of the year $\left(\vec{x}_{j}, s_{j}\right)$ are indicated.

7. Rec de Romanyá. Altitud: $70 \mathrm{~m}$.

8 y 9 . Rec del Jonquet. Altitud: 30 m. y 25 m.. respectivamente.

10 y 11 . Rec d'Escollasa. Altitud: 20 m. y 10 m., respectivamente.
12. Rec de Joncols. Altitud: $25 \mathrm{~m}$.

Las estaciones 2, 3 y 6 están emplazadas sobre un lecho arenoso, llano y bastante amplio. El resto están ubicadas en vaguadas generalmente angostas, de pendiente acusada y sustrato rocoso; el lecho es irregular 


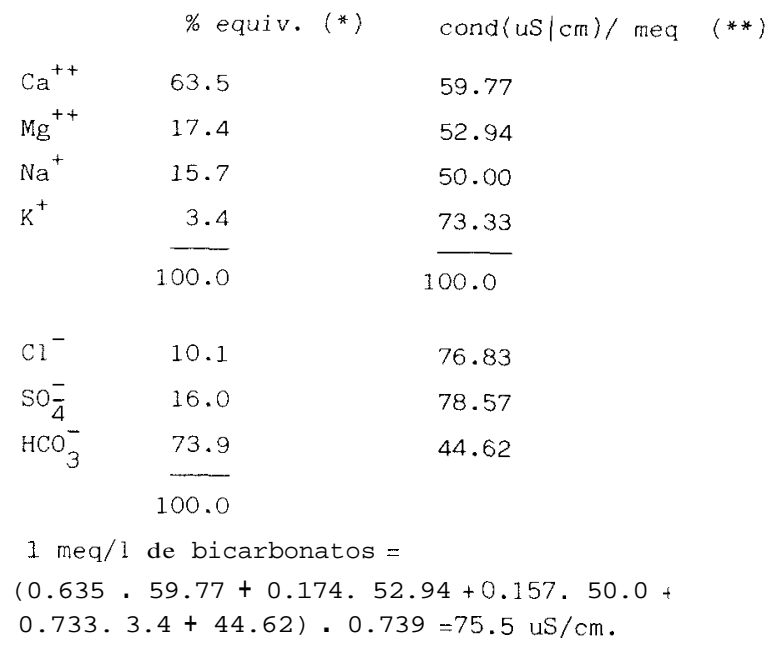

Tabla 3.-Proporciones relativas de los iones principales (en equivalentes)en un agua dulce «armónica»(cf. Margalef 1955 b) y su contribución a la conductividad total $(\mathrm{en} \mu \mathrm{S} / \mathrm{cm} /$ meq);se indica además la conductividad teórica de un agua con una alcalinidad de 1 meq/l. (*)segúnMargalef 1983.(**) a partir de Golterman et al. (1978).

Ratio of the main ions (in equivalents) of an «harmonic» water (cf. MARGALEF 1955 b), and their contribution to the conductivi ty (in $\mathrm{US} / \mathrm{cm} / \mathrm{meq}$ ). The theoric conductivity ofa water with an alkalinity of $1 \mathrm{meq} / \mathrm{l}$ is also indicated. $\left(^{*}\right)$ from MARGALEF 1983. $\left.{ }^{* *}\right)$ from GOLTERMAN e’ al 1978.

\begin{tabular}{crrrr} 
& \multicolumn{3}{c}{$\mathrm{mg} \mathrm{l^{-1 }}$} \\
& $\mathrm{Na}^{+}$ & \multicolumn{1}{c}{$\mathrm{K}^{+}$} & $\mathrm{Ca}^{2+}$ & $\mathrm{Mg}^{2+}$ \\
$\mathrm{IX}-84$ & 134.3 & 38.5 & 148.1 & 54.2 \\
XI-84 & 101.5 & 8.4 & 3.2 & 14.2
\end{tabular}

Tabla 4.-Concentración de los cationes principales en la estación 8 en setiembre y noviembre de 1984 .

Concentration ofmajor cations in station 8 in Septemberand November 1984

y está formado por superficies más o menos lisas, alternando con cubetas de dimensiones variables.

Los materiales básicos para este estudio se obtuvieron en cuatro salidas de campo: 17-18 de febrero, 11-12 de mayo, 14 de setiembre y 29-30 de noviembre de 1984.

Generalmente, se tomaron muestras in situ de temperatura (termómetro de alcohol), $\mathrm{pH}$ (con un $\mathrm{pH}-$ metro Crison 506) y conductividad (con un conductímetro Crison 523) del agua. La alcalinidad se midió en el laboratorio, por valoración con $\mathrm{H}_{2} \mathrm{SO}_{4}(0.02 \mathrm{~N})$ $\mathrm{y}$ un indicador mixto.

Paralelamente, se recogieron muestras de algas, procedentes de diversos hábitats (herpon, plocon, pecton, etc.), que se fijaron con unas gotas de formaldehído al $40 \%$. Una parte de cada muestra se reservó para la observación directa al microscopio óptico. La otra parte fue previamente tratada con $\mathrm{H}_{2} \mathrm{SO}_{4}$ concentrado y KNO, (segun la técnica descrita por Hustedt, 1930), con el fin de eliminar la materia orgánica de los frustulos de las diatomeas y facilitar su determinación. Los frústulos limpios se montaron en preparaciones permanentes, utilizando una resina sintética (Naphrax, I.R.: 1.74). De cada preparación de algas se elaboró una lista taxonómica completa. En las diatomeas se estimó, además, la abundancia relativa de las especies mediante los símbolos ya conocidos 5 , $4,3,2,1 \mathrm{y}+$.

\section{RESULTADOS Y DISCUSION}

La mayor parte de las aguas continentales del Cabo de Creus se localizan en pequeños torrentes de poco caudal, escasa profundidad y sustrato silícico. Estos sistemas son, por sus características, muy sensibles a las condiciones del entorno.

La temperatura del agua, por ejemplo, refleja con bastante fidelidad la evolución térmica del aire, a escala diaria y a lo largo del año (tabla 1), presentando una oscilación media anual de $13,5^{\circ} \mathrm{C}$.

Las variaciones del resto de los parámetros medidos pueden hallarse en la tabla 2.

El pH es poco estable, fluctuando desde valores ligeramente ácidos en febrero (alrededor de 6.5) hasta otros más básicos en el resto del año, especialmente en setiembre. Estas fluctuaciones, así como la aparición de medidas anormalmente altas (8.1-9.2) para aguas que corren por terrenos esquistosos, hay que interpretarla en función de la alcalinidad y temperatura del agua, y de la intensidad de los procesos productores y consumidores de $\mathrm{CO}$, (fotosíntesis, respiración, etc.), estos últimos no controlados en este estudio.

La alcalinidad es moderada casi siempre (con promedios estacionales cercanos a $1 \mathrm{meq} / \mathrm{l}$ ) excepto en setiembre, momento en el cual se produce un aumento importante de este parámetro en gran parte de las localidades.

La conductividad es muy alta, si se compara con la de otras zonas silicicas de Cataluña. La media de los valores registrados es de $1147 \mu \mathrm{S} / \mathrm{cm}$, con un rango de variación entre 270 y $4041 \mu \mathrm{S} / \mathrm{cm}$. Generalmente, los máximos corresponden a febrero y setiembre.

En todas las estaciones la relación alcalinidad/conductividad (A/C) es inferior a la que correspondería a un agua dulce «armónica» (1/75.5), entendiéndose 
TABLA V. Evolución temporal de las comunidades de algas en las estaciones estudiadas. p=plocon, pe=pecton, h=herpon y pl=plancton. TABIE V. Temporal evolution of the algal communities in the studied stations. $p=p l o c o n$, pezecton, $h=h e r p o n$ and $p l=p l a n c t o n$.

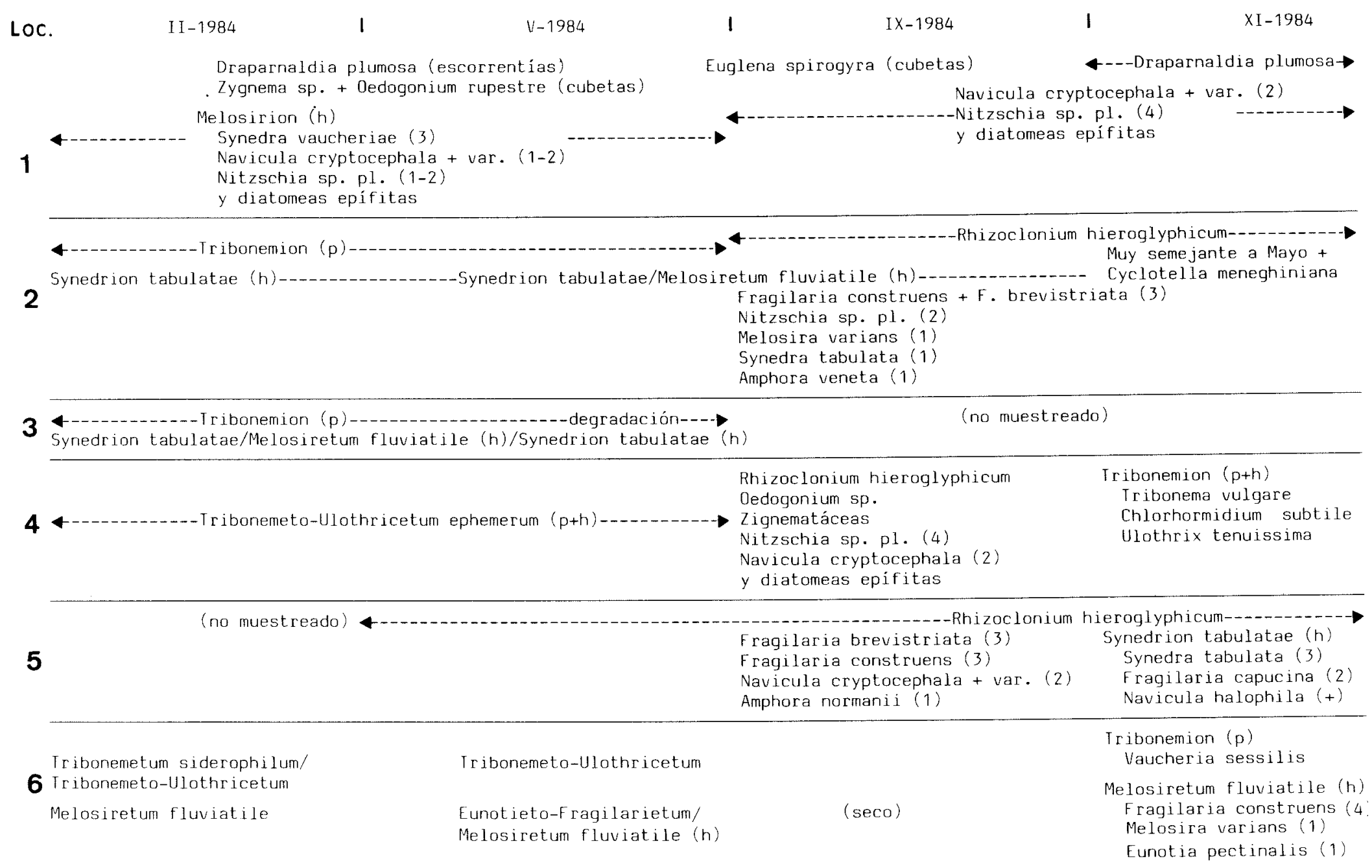


- Tribonemetum siderophi lum Nitzschia sp. pl. (3) (cubetas) (cubetas) Bacterias del hierro
Fragilaria construens (1) Navicula radiosa (1)
Melosiretum rivulare $p+h$ Draparnaldia plumosa Amphipleura pellucida

Ir ibonemeto-Ulothricetum/

Tribonemetum siderophilun
84

8 - Iribonemeto-Ulothricetum ( $p$ Melosiretum fluviatile (h)

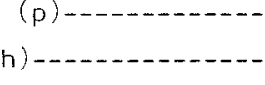
helomonas hi Nitzena proxima Navicula cryptocephala (2)

Melosiretum fluviatile (h) Synedra vaucheriae (3) Melosira varians (1) Fragilaria capucina (1) Eunotia pectinalis $(+)$

Cladophorion/Tribonemion ( $p$ ) Cladophora fracta Phacus acuminatus

9 (no muestreado)

Peridinium pusillum

Pediastrum boryanum

Melosiretum fluviatile (h) Nitzschia sp. pl. (1) Navicula cryptocephala (1 Epithemia zebra (4)
Cladophorion $(p)$ Cladophora fracta Rhizoclonium hierogly. Chara globularis

Melosiretum fluviatile (h) Melosira varians (1) Fragilaria capucina (1) Eunotia pectinalis (1)

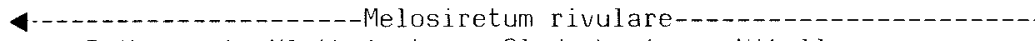

10 ----Tribonemeto-Ulothricetum + Cladophorion + Nitella opaca--

-Cladophorion--.----on-

Iribonemion $(p+h)--.----$ Rhizoclonium

4-Diatomeas halófilas $(+)$. (Amph. veneta, Syn. pulchella, S. tabulata)

11

-Melosiretum rivulare ( $p+p e)-$ Hildenbrandietum rivulare ( $p+p e$ ) Diatomeas epífitas y halófilas $(+)$ Cladophorion $(p)$

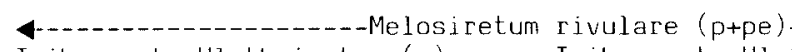
Tribonemeto-Ulothricetum $(p)$

Tribonemeto-Ulothricetum ribonemetum siderophilum

12

Nitzschia sp. pl.

Synedra vaucheriae (2)

Melosira varians (1)

Navicula cryptocephala (1)
Nitzschia sp. pl. (3)

Navicula cryptocephala (2)

Rhopalodia gibba (2)

Epithemia zebra (1)
Melosiretum rivulare $(p+h)$ Draparnaldia plumosa Amphipleura pellucida Iribonemeto-Ulothricetum $(p+h)$

Iribonema sp. pl.

Vaucheria sessilis Chlorhormidium subtile 
por tal un agua que tuviera por composición iónica relativa la que podría considerarse como promedio de las aguas dulces de todo el mundo (cf. Margalef 1955, pág. 20) (tabla 3). Esto hace pensar que, en los sistemas estudiados hay un exceso de sales neutras en disolución.

Atendiendo a la composición mineralógica del Cabo de Creus, estas sales sólo pueden proceder del mar y, por lo tanto, han de ser en su mayoría cloruros. La influencia marina se produce principalmente por vía eólica, ya que una entrada directa de agua de mar sólo es posible en un número reducido de localidades (2, 3 y, excepcionalmente, 4 y 5). La existencia de este transporte aéreo es evidente a simple vista; la sal cristaliza sobre las rocas y sobre los vegetales, inhibiendo el crecimiento de sus partes más expuestas.

En la figura 2 se puede apreciar que, en las localidades más próximas al mar o en las menos resguardadas (estaciones 2, 4 y, en menor medida la 10), la influencia marina es muy importante y fluctuante en el tiempo. Estas fluctuaciones afectan fundamentalmente a la conductividad, de manera que los segmentos de recta que unen los puntos de una misma estación muestran pendientes reales muy bajas $(\triangle A / \triangle C \simeq 0)$.

En las estaciones 1, 8 y 12, la influencia del mar es menor y, a la vez, menos variable a lo largo del año (sólo en febrero es sensiblemente superior). Tampoco es muy variable la alcalinidad, si exceptuamos el máximo espectacular de verano. En este último caso, la razón de incrementos $\triangle \mathrm{A} / \triangle \mathrm{C}$, entre mayo y setiembre alcanza valores relativamente próximos a $1 /$ 74 , lo que indica que estos incrementos se deben, sobre todo, a un aumento de la concentración de sales alcalinas (p. eje., bicarbonatos) en el agua.

El análisis de los cationes principales en una de estas localidades (estación 8), en setiembre y noviembre, confirma la anterior afirmación (tabla 4). Efectivamente, el pico de la alcalinidad en setiembre coincide con una concentración muy elevada de $\mathrm{Ca}^{2+}$, el catión que más contribuye a la alcalinidad en las aguas dulces, ligado al anión bicarbonato. Descartada la posibilidad de que este calcio provenga del mar (por comparación de las concentraciones de $\mathrm{Na}+\mathrm{y}$ $\left.\mathrm{Ca}{ }^{2+}\right)$ o de que sea fruto de la alteración de las rocas de la región, sólo queda la alternativa de suponer que su origen es la descomposición de materia orgánica, especialmente algas filamentosas, que tiene lugar con gran intensidad entre primavera y verano.

La estación 7 es la más alejada del mar y muestra un ciclo particular, en el que no se observan grandes fluctuaciones. Del resto de estaciones $(3,5,6,9$ y 11$)$ sólo se dispone de datos aislados o incompletos.

Los resultados del estudio de las comunidades de algas se resumen en la tabla 5. En la mayoría de los casos, los complejos de especies hallado se han podido incluir en unidades fitosociológicas bien definidas (alianzas o asociaciones). En aquéllos en que no ha sido posible, se da una breve relación de las especies más abundantes o características.

Generalmente, las biocenosis no están compuestas por una única asociación sino por varias, que a menudo representan las comunidades de algas que viven en residencias ecológicas distintas (plocon, herpon, etc.). Para distinguir las unas de las otras, junto al nombre de cada alianza, asociación o lista de especies se ha indicado, entre paréntesis, a que tipo de comunidad subordinada representa $(\mathrm{p}=\mathrm{plocon}, \mathrm{h}=\mathrm{herpon}$, pe=pecton).

Como base para la caracterización y denominación de las unidades biocenológicas se ha utilizado la clasificación de Margalef (1983), que funde, con pequeñas modificaciones, los modelos presentados en varias publicaciones anteriores.

Desde este punto de vista, el Cabo de Creus debe considerarse como dominio de la Tribonemetalia, como ya indicara Margalef (1955b) en un esbozo de la limnología regional de Cataluña.

Sobre lechos rocosos, donde el agua circula con rapidez, suele desarrollarse el Melosiretum rivulare (con Lemanea fluviatilis, Draparnaldia plumosa y, en algunos casos, Compsopogon sp.), que puede ir acompañado del Hildenbrandietum rivulare (Al. Hydrococcion).

Las especies características del Tribonemion configuran, gran parte del año, el plocon de las zonas más profundas, con menor corriente. Cuando es posible diferenciar las asociaciones de esta alianza, se observa que el Tribonemeto-Ulothricetum ephemerum (caracterizado, entre otras, por especies de Tribonema, Oedogonium Vaucheria, Hormidium y, en ocasiones, Ulothrix) prospera en condiciones de mayor renovación del agua. El Tribonemetum siderophilum (con Tribonema, Oedogonium, Epithemia, Ophiocytium y Apiocystis brauniana) y, en última instancia, poblaciones de euglenales, caracterizan la evolución hacia estadios de mayor estanqueidad.

Las comunidades de diatomeas pueden ser parte de las asociaciones anteriores, pero lo usual es que tengan su propia identidad, constituyendo casi siempre un Melosiretum fluviatile (con Melosira varians como especie dominante, Eunotia pectinalis, Rhopalodia gibba, etc.). En las estaciones más próximas al mar, donde la conductividad puede ser especialmente alta, el $M$. fluviatile se desfigura por la pérdida de especies diagnóstico (E. pectinalis, R. gibba) y por la aparición de otras (Navicula halophila, Synedra tabulata, Thalassiosira weissflogii, e incluso filamentosas, como Oedogonium capilliforme), que pueden considerarse como características de la Al. Synedrion tahulatae.

A estos elementos que definen la pertenencia de las comunidades a una u otra unidad fitosociológica, se añade un fondo común de especies halófilas y fre- 
cuentes en ambientes subaéreos, que confirman la presencia notable de cloruros en el agua y el carácter temporal de los sistemas estudiados. Son muy habituales, por ejemplo, Achnanthes delicatula, A. lanceolata, A. rostrata, Amphora veneta, Hantzschia amphioxys, Navicula cincta, N. contenta, N. gregaria, $N$. halophila, N. minina, N. minuscula, N. seminulum, Nitzschia vitrea, Rhoicosphenia curvata (=R. abbreviata), Synedra pulchella y $S$. tabulata, entre otras. La existencia en la mayoría de las localidades, de algas filamentosas de pared celular desnuda (Oedogonium, Rhizoclonium y, a veces, Cladophora) contribuye, aún más, a incrementar la complejidad de las poblaciones, aportando cantidades más o menos importantes de diatomeas epífitas, como Achnanthes minutissima, Cocconeis placentula, Epithcmia sp. pl., Gomphonema sp. pl. y Synedra ulna.

El máximo desarrollo de las comunidades de algas (en biomasa y variedad de especies) se produce desde finales de invierno hasta principios de verano. En este tiempo, la mayoría de clorofíceas filamentosas alcanzan la fertilidad y maduran las zigósporas.

En verano, algunas estaciones se secan por completo. En las demás la fisionomía de las comunidades cambia rotundamente. Desaparece el Melosiretum rivulare y el Tribonemion alcanza su facies terminal (formada por poblaciones de euglenales) o deja de existir. Las poblaciones de diatomeas también se modifican; desaparecen muchas de las especies abundantes en otros períodos del año, incrementándose, en cambio, la representación de especies comunes en aguas eutróficas o con mucha materia orgánica (p. ej., Navicula cryptocephala, Nitzschia frustulum, N. pala, N. paleacea. N. microcephala, etc.). Rhizoclonium hieroglyphicum que generalmente está arrinconado en las zonas más salobres, en verano invade otras partes. Cladophora glomerata var. crassior, otro elemento del Cladophorion, sólo le acompaña en los lugares donde el agua es más persistente y el aumento de la alcalinidad es más notable.

En otoño, las comunidades comienzan a recuperarse de nuevo. Aparecen las especies que caracterizan las facies iniciales de cada asociación: Draparnaldia plumosa y Amphipleura pellucida por el Melosiretum rivulare, Tribonema sp. pl. por el Tribonemion y Melosira varians, Eunotia pectinalis y Fragilaria capucina por el Melosirion.

\section{AGRADECIMIENTOS}

Los autores agradecen al Dr. R. Vallejo (Cátedra de Edafologia de la Univ. de Barcelona) la ejecución del análisis de los cationes principales y sus comentarios sobre los resultados obtenidos. A Ana M. ${ }^{a}$ Domingo la realización de las figuras.

\section{BIBLIOGRAFIA}

CARRERAS, J. 1974. Petrologia y análisis estructural de las rocas metamórficas de la zona del Cabo de Creus (Prov. de Gerona). Resumen Tesis Doctoral. Secret. Public. Interc. Científico y Ext. Univ. Universidad de Barcelona. 11 págs.

GOLTERMAN, H. L.; CLYMO, R. S. y OHNSTAD, M. A. M. 1978. Methods for physical and chemical analysis of fresh waters. 2." Ed. I.B.P. Handbooks n. ${ }^{\circ}$ 8. Blackwell Scientific Publications. Oxford. 213 págs.

HUSTEDT, F. 1930. Bacillariophyta (Diatomeae). In: Pascher. Süsswasser-Flora Mitteleuropas, 10. Gustav Fischer. Jena. 466 págs.

MARGALEF, R. 1949. Materiales para una flora de las algas del NE de España. Euchlorophyceae. Collectanea Botanica, 2(2):233-250.

MARGALEF, R. 1950. Materiales para una flora de las algas del NE de España. Euchlorophyceae. Collectanea Botanica, 2(3):273-293.

MARGALEF, R. 1953. Materiales para una flora de las algas del NE de España. Cyanophyceae. Collectanea Botanica, $3(3): 231-260$.

MARGALEF, R. 1954. Materiales para una flora de las algas del NE de España. Bacillariophyta. Collectanea Botanica, 4 (1): 53-79; 4 (2): 183-201.

MARGALEF, R. 1955a. Materiales para una flora de las algas del NE de España. Desmidiales, Rhodophyceae. Collectanea Botanica, 4 (3):319-330.

MARGALEF, R. 1955b. Los organismosindicadores en la limnologia. Biologia de las aguas continentales, 12. Ministerio de Agricultura, Dir. Gral. de Montes, Caza y Pesca. Madrid. 300 págs.

MARGALEF, R. 1958. Materiales para el estudio de las comunidades de aguas dulces y salobres, principalmente del NE de España. Publ. Inst. Biol. Apl., 28: 5-47.

MARGALEF, R. 1983. Limnologia. Omega. Barcelona. 1010 págs. 